\title{
Reading and reacting to faces, the effect of facial mimicry in improving facial emotion recognition in individuals with antisocial behavior and psychopathic traits
}

\author{
Melina Nicole Kyranides ${ }^{1}\left[\right.$ ] Maria Petridou $^{2} \cdot$ Heema Ajeet Gokani $^{1} \cdot$ Sam Hill ${ }^{1} \cdot$ Kostas A. Fanti $^{2}$
}

Accepted: 19 January 2022

(C) The Author(s) 2022

\begin{abstract}
Individuals with antisocial behavior and psychopathic traits are characterized by deficits in processing facial expressions, which results in poor social adaptation and dysfunctional interpersonal relationships. However, it is not clear how individuals with varying levels of these traits differ in this emotional impairment and if these deficits are due to correctly identifying or reacting appropriately to facial expressions. The aim of the study was to examine how individuals with these traits recognized and responded to affective facial expressions by using FaceReader software (which analyses footage of faces) across different experimental conditions (imitation, suppression, and control). Imitating facial expressions was introduced to examine whether it could direct participants' attention to the facial cues and improve participants' performance on the facial task. A total of 643 individuals from the community were pre-screened and a sample $(N=107 ; M$ age $=21.08, S D=1.55)$, differentiated on levels of antisocial personality disorder (APD) symptoms and psychopathic (PSY) traits, who were selected based on extreme scores (high/low), were invited to participate in the study. Individuals with higher levels of APD symptoms and PSY traits (APD + PSY) expressed more anger than other groups, while those in the APD-only group expressed more sadness, compared to other groups. Overall, participants were compliant in following the instructions to imitate facial expressions. However, only the group with predominantly APD symptoms and the group with combined symptoms (APD + PSY) showed improvement in their accuracy ratings specifically when instructed to imitate facial expressions, compared to when no instructions were provided. The study offers a promising direction for targeting deficits in facial emotion recognition, suggesting that the deficits found in individuals with behavioral problems (with and without psychopathic traits) can be improved by asking them to imitate facial expressions.
\end{abstract}

Keywords Psychopathic traits $\cdot$ Antisocial behavior $\cdot$ Facial emotion recognition $\cdot$ Facial expressions $\cdot$ FaceReader

Melina Nicole Kyranides

melina.nicole.kyranides@ed.ac.uk

Maria Petridou

petridou.maria@ucy.ac.cy

Heema Ajeet Gokani

H.Ajeet-Gokani@sms.ed.ac.uk

Sam Hill

s.h.hill@sms.ed.ac.uk

Kostas A. Fanti

kfanti@ucy.ac.cy

1 Department of Clinical and Health Psychology, School of Health in Social Sciences, The University of Edinburgh, Medical School (Doorway 6), Teviot Place, EH8 9AG Edinburgh, UK

2 Department of Psychology, The University of Cyprus, Nicosia, Cyprus

\section{Introduction}

Antisocial personality disorder (APD) and psychopathy represent two overlapping constructs with associations to severe antisocial traits (Anton et al., 2012; Crego \& Widiger, 2015). APD is conceptualized in the Diagnostic and Statistical Manual of Mental Disorders - Fifth Edition (DSM5; American Psychiatric Association, 2013) as primarily a behaviorally based construct with an emphasis on antisocial behavior. Psychopathy on the other hand is only included in the DSM-5 as a specifier for APD, but theoretical models of the disorder emphasize personality traits rather than behavior. Psychopathy includes personality dispositions according to the triarchic model (i.e., boldness, meanness, and disinhibition), which encompass affective, interpersonal, and behavioral features (Patrick et al., 2010). The interpersonal 
and affective features of psychopathy have been shown to be differentially related to APD, further distinguishing the two constructs (Anton et al., 2012; Venables et al., 2014). The diagnostic criteria for APD, therefore, do not always include psychopathic traits (Crego \& Widiger, 2015; Venables et al., 2014), which should be taken into account as the presence of these traits has been associated with a worse prognosis (Riser \& Kosson, 2013).

Correctly interpreting and conveying affective states is crucial for social cognition and healthy social functioning (Blair, 2018) and has been found to be affected in individuals with both behavioral problems and psychopathic traits (Marsden et al., 2019). Facial expressivity plays a central role in interpersonal relationships, as it communicates silent social cues and reinforces socially acceptable behaviors. While deficits in emotion processing (e.g., lack of empathy) are addressed in conceptualizations of APD, it is possible to meet diagnostic criteria based on the prevalence of antisocial behavior alone (Sarkar et al., 2011). Emotional dysfunction, however, is central to a diagnosis of psychopathy and is, therefore, thought to differentiate those with psychopathy from those with APD (Sarkar et al., 2011). Prior studies suggest that individuals with psychopathic traits are characterized by deficits in facial emotion recognition and facial expressivity, which in turn results in poor social adaptation and dysfunctional interpersonal relationships (Blair, 2018; Kyranides et al., 2020). It is unclear whether similar deficits observed in APD are symptomatic of the disorder or are rather a result of comorbid psychopathic traits (Marsden et al., 2019). The present study aimed to further differentiate emotion processing deficits in individuals with these traits by examining how individuals with isolated or co-occurring APD symptoms and psychopathic traits (i.e., APD-only, PSY-only, and APD + PSY): a) identify/recognize affective facial expressions and b) comply to instructions asking them to imitate facial expressions. The study adds to prior work by determining whether an instruction to imitate facial expressions improves facial emotion recognition in individuals with these traits by directing their attention to the facial features displayed by asking them to experience the emotion through imitation.

\section{Antisocial Behavior, Psychopathy and Facial Emotion Recognition}

Empirical evidence suggests that individuals with psychopathic traits show deficits in facial emotion recognition (Dawel et al., 2012; Marsh \& Blair, 2008). The relationship is well-established in demonstrating specific deficits for fearful and sad expressions (Dadds et al., 2008; Fairchild et al., 2009), but some studies indicate a general impairment in recognition across emotional expressions (Dawel et al., 2012; Kyranides et al., 2020). While some research suggests links between APD and facial emotion recognition deficits in antisocial populations (i.e., Marsh \& Blair, 2008; Rogstad \& Rogers, 2008), few have considered APD with or without comorbid psychopathic traits. For example, Bagcioglu et al. (2014) found more severe deficits in recognizing disgust in an APD sample compared to controls, and Habel et al. (2002) found deficits in correctly identifying happy and sad facial expressions, but neither study controlled for psychopathic traits in their samples. Conversely, a comparison of individuals with APD without psychopathy showed no differences compared to controls in a facial emotion recognition task (Schiffer et al., 2017). With regard to sadness, individuals displaying antisocial behavior and psychopathic traits were found to exhibit greater impairments compared to those with antisocial behavior alone (e.g., Fairchild et al., 2009). A recent meta-analysis of facial emotion recognition findings concluded that samples with APD with comorbid psychopathy showed increased difficulty processing negative affective stimuli, while samples with APD only, without psychopathy, showed no such deficits (Marsden et al., 2019). Findings from work in facial emotion recognition further demonstrate that antisocial behavior combined with psychopathic traits represents a separate subgroup (Kyranides et al., 2016, 2020; Verona et al., 2012) of individuals with different emotional deficits.

\section{Neurocognitive Models of Emotion Processing}

Deficits in facial emotion processing in individuals with psychopathic traits are thought to reflect deficits in attention processing (Newman et al., 2010). In the response modulation model, Patterson and Newman (1993) suggest that individuals with psychopathic traits experience an attentional bias during goal-directed behavior, which hinders their ability to reallocate attention to peripheral information secondary to their goals. Experimental studies have shown that individuals with psychopathic traits are more prone to ignore potentially meaningful peripheral information once engaged in goal-directed behavior (MacCoon et al., 2004), even when that information may be threat-related (e.g., electric shock) (Baskin-Sommers et al., 2012). The model is thought to extend to social interactions in which the attention deficit may moderate affective deficits seen in individuals with psychopathic traits (Baskin-Sommers et al., 2012; Patterson \& Newman, 1993). In social interaction, the affective state of a counterpart may be peripheral to an individual's goals and thus ignored. Abnormal processing of affective information may explain why individuals with psychopathic traits find it easier to engage in behavior that causes another person harm (Blair, 2018). If affective deficits are moderated by attention deficits, then a manipulation of attention to affective information could improve emotion processing 
abilities of individuals with psychopathic traits. Following this rationale, the current study aims to investigate this possibility by making the affective information more central to the participant.

When observing facial expressions, typically developing individuals experience partial activation of the neural representation that corresponds to the experience of the emotion being observed in others, which is then thought to aid in emotion processing such as facial emotion recognition (Prochazkova \& Kret, 2017; Wood et al., 2016). This process of vicarious neural activity appears to be impaired in individuals with high psychopathic traits (Lockwood, 2016; Seara-Cardoso et al., 2016), which has led some researchers to propose a cognitive model of antisocial behavior based on impaired learning through aversive conditioning (Blair, 2013). For example, when confronted with aversive stimuli such as fearful facial expressions, individuals with psychopathic traits may fail to associate the aversive feedback (i.e., distress of victim) with their own behavioral transgressions and thus not learn the repercussions of their aggressive behavior (Blair, 2013). According to the response modulation model, this failure to associate transgression with outcome can be explained by the individual not attending to the relevant affective information (i.e., facial expression), as it is peripheral to their goals.

Theoretical models of empathy emphasize the importance of embodiment, i.e., experiencing another's affective state, which helps in relating to and understanding that affective state (Bird \& Viding, 2014). Embodiment is thought to promote understanding of an affective state through a mechanism of emotional contagion in which the observation of another's emotion causes the observer to share the same affective state (Prochazkova \& Kret, 2017). In their shared-representation theory of social cognition, Sommerville and Decety (2006) suggest that since the perception and experience of emotion draw on the same neural circuitry, an observer must be able to experience an emotion to perceive it in others. In such models, emotional contagion is not sufficient for empathy, but it is necessary, and it can only occur if an individual can embody an emotional experience. Goldman and Sripada (2005) showed that deficits in facial emotion recognition are associated with deficits in the ability to produce the same emotion. Individuals with psychopathic traits seem to experience emotions differently (Bird \& Viding, 2014) and, in particular, display more restricted physiological reactions when presented with fearful stimuli (Kyranides et al., 2016, 2017). According to their self to other model of empathy, Bird and Viding (2014) argue that individuals with high psychopathic traits may not be able to interpret fear in others because they are not capable of experiencing fear and possibly other emotions in the same way.

\section{Antisocial Behavior, Psychopathy and Facial Expressivity}

Typically developing individuals engage in automatic facial mimicry when observing the expressions of others (Chartrand \& Bargh, 1999; Hess \& Blairy, 2001). Facial electromyography (fEMG) has proven to be a useful methodology in demonstrating such isomorphic facial reactions through the direct measure of electrical changes in facial muscles (Hess \& Blairy, 2001; Niedenthal et al., 2000). Furthermore, the prevalence of facial mimicry has been positively associated with empathy (Chartrand \& Bargh, 1999). However, the findings regarding facial mimicry in individuals with psychopathic traits are varied; for instance, Book et al. (2015) showed that individuals with psychopathic traits had intact ability to accurately mimic the emotional expression of fear and remorse, but additional work assessing mimicry in a nonclinical population found that individuals with high psychopathic traits had difficulties reflecting negative emotions in others (e.g., anger, fear, disgust, or sadness) but not positive emotions (e.g., happiness and smiles) (Khvatskaya \& Lenzenweger, 2016; Owens et al., 2017). In contrast, Künecke et al. (2018) found no differences in facial reactivity between psychopathic offenders and a control group.

Studies looking at facial responsiveness in individuals with disruptive behavior using fEMG have shown weaker facial responses to affective cues (de Wied et al., 2012). When assessing sadness and happiness, studies found that adolescents with disruptive behavior and high callous unemotional traits (the precursor of psychopathic traits) showed reduced facial reactivity to affective cues (de Wied et al., 2006, 2012). Conversely, Fanti et al. (2017) found that individuals high on impulsive aggression produced more angry facial expressions when viewing violent films, and recent evidence suggests that individuals with psychopathic traits tend to express more anger (Kosson et al., 2020). Inconsistent findings and a lack of research around facial mimicry in community samples with psychopathic traits and APD symptoms require further investigation, which will be addressed in the current study.

Deficient facial mimicry in populations with deficits in empathy may be related to differences in neural activity during empathizing (Fecteau et al., 2008). Vicarious neural activity in the observation of emotional expressions is thought to underlie empathy, and the Mirror Neuron System (MNS) could serve as the basis for such activity (Gazzola et al., 2006). Simultaneous measurement of facial muscular reactions using fEMG and neural responses (i.e., Blood Oxygenation Level Dependent [BOLD] responses) using functional magnetic resonance imaging (fMRI) suggests that facial mimicry is related to activity in the MNS during emotion processing (Rymarczyk et al., 2018). MNS dysfunction has been implicated in empathic deficits in 
psychiatric disorders, especially autism spectrum disorders (ASD) (e.g., Dapretto et al., 2006), but few have directly investigated impaired MNS activity during emotion processing in psychopathy or APD. Fecteau et al. (2008) found that decreased MNS activity was correlated with the interpersonal and affective dimensions of psychopathy but not to antisocial behavior. Meffert et al. (2013) found that participants with high psychopathic traits exhibited reduced spontaneous vicarious neural activity when viewing videos depicting affective touch. However, they also demonstrated that deficits were significantly reduced when participants were instructed to empathize with the actors in the videos, suggesting that individuals with psychopathy may be capable of near normal activation when empathizing is deliberate. In accordance with cognitive models of empathic deficits in psychopathy (e.g., Bird \& Viding, 2014), this evidence indicates that individuals with psychopathic traits may be able to restore emotional understanding, although incompletely, through mentalizing. Indeed, Meffert et al. (2013) found that the increase in activity in brain regions of interest was accompanied by increases in regions associated with mentalizing (i.e., medial prefrontal cortex and the temporoparietal junction).

\section{Interventions Addressing Facial Emotion Recognition Deficits}

While interventions in emotion processing in individuals with behavioral problems and psychopathic traits have remained elusive (Chialant et al., 2016), evidence from therapeutic efforts in this area suggests that facial emotion recognition abilities can be improved. The eye region of the face has been shown to be especially important in deciphering emotions in others (Lee \& Anderson, 2017), and, when typically developing individuals are asked to make judgements about an emotion being expressed, they tend to fixate on the eye region (Peterson \& Eckstein, 2011). Prior research also shows that individuals with psychopathic traits show reduced attention to the eyes (Gehrer et al., 2020; Gillespie et al., 2015), which is thought to contribute to emotion recognition deficits in this population (Dadds et al., 2008). Dadds et al. (2008) were able to demonstrate that, at least in children, these deficits can be temporarily reversed by a simple verbal instruction to fixate on the eyes. More recently, Kyranides et al. (2020) found improvements in facial emotion recognition accuracy when using a silent cue manipulation to redirect attention to various parts of the face, but improvements were not specific to one region of the face. More studies are needed to understand how interventions might differentially affect individuals with antisocial behavior and psychopathic traits.

\section{Current Study}

The main purpose of this study was to examine how individuals with isolated or co-occurring APD symptoms and psychopathic traits (i.e., APD-only, PSY-only, and APD + PSY) process facial stimuli by examining their a) facial emotion recognition accuracy and b) facial reactions when presented different facial expressions. Additionally, c) the study examined whether providing different instructions to participants (imitation, suppression) would affect participants' performance on a facial emotion recognition task. FaceReader was used to assess whether the instructions to mimic and suppress were followed by participants and to address some of the limitations of using fEMG (e.g., unclear discrimination between distinct facial expressions such as sadness and anger). Based on prior work (Fairchild et al., 2009; Marsh \& Blair, 2008), it was hypothesized that facial emotion recognition accuracy would be negatively associated with psychopathic traits and APD symptoms, although differences between groups were not predicted due to the inconsistent findings (Marsden et al., 2019). It was expected that the group with co-occurring APD and psychopathic traits would present more deficits compared to all other groups, due to their more severe deficits. Based on prior work on expressivity in response to affective facial expressions (de Wied et al., 2006, 2012), it was hypothesized that individuals with antisocial behavior would exhibit reduced expressivity across emotions, while individuals with psychopathic traits were expected to show decreased expressivity for negative emotions (i.e., anger, fear, and sadness) but not positive emotions (i.e., happiness) (Khvatskaya \& Lenzenweger, 2016; Owens et al., 2017). The current study also used an instruction to imitate facial expressions to manipulate attention and examine if this improved facial emotion recognition. The explicit instruction to imitate facial expressions is expected to improve facial emotion recognition based on similar findings in previous studies with clinical (e.g., autistic traits; Lewis \& Dunn, 2017) and nonclinical populations (Conson et al., 2013; Schneider et al., 2013), while inhibiting facial mimicry has been found to impair facial emotion recognition (Neal \& Chartrand, 2011; Oberman et al., 2007). The present study will build on previous work by comparing how groups with varying profiles of APD symptoms and psychopathic traits differentially benefit from an instruction to imitate.

\section{Method}

\section{Participants}

Data were collected originally from 643 young adults (141 males; $M$ age $=21.10, S D=2.22$ ) recruited from 
different departments of the University of Cyprus who responded to an online advert. The questionnaire package was administered individually online through a secure internet-based survey platform. The initial sample $(N=643)$ was diverse in terms of family status: $68.3 \%$ were single, $17.1 \%$ were in a relationship, $3.1 \%$ were married, $0.3 \%$ were divorced, and $11.2 \%$ characterized their relationship status as "other". Participants presenting extreme scores (high and/or low based on the mean obtained from the initial sample) on the measures assessing APD symptoms and/or psychopathic traits were selected and invited to participate in the experimental phase of the study (more information in the results section). Pre-selection of participants was carried out to ensure the inclusion of individuals with extreme scores with varying profiles of APD symptoms and psychopathic traits. The total number of participants recruited to participate in the experimental phase was 110 , although the analysis was only carried out with 107 , as three participants' data were lost due to technical issues. Of the 107 participants $(M$ age $=21.08, S D=1.55), 46$ (31 females) made up the low-risk group, 21 (14 females) the PSYonly group, 17 (12 females) the APD-only group, and 23 (16 females) the combined APD + PSY group (see results for group differences). Before participating in the experimental phase of the study, the screened participants were asked if they had a history of epilepsy or any other serious mental or medical issues. None were reported. Participants received course credit for their participation.

\section{Procedure}

All participants in the experimental session were tested individually. After reading the information sheet, participants provided informed consent. The timing of events, the presentation of stimuli, and the logging of participants' responses were controlled by an E-prime script (E-prime 3.0). The experimental task was presented on a laptop. Following the presentation of each stimulus, participants entered their responses by correctly identifying the emotion being displayed by pressing a key on the keyboard with the number that corresponded to the emotion. Participants were asked to refrain from covering their faces during the task, as they were informed that their facial expressions would be recorded during the experiment with a camera that was placed on the computer screen at eye level. Recordings were edited and processed offline with FaceReader, and only the 5-s sections during and after the stimuli presentation were analysed. The approximate duration of the computerized task was $10 \mathrm{~min}$. After completing the task, participants were debriefed and thanked for their time.

\section{Measures}

\section{Adult Self-Report Inventory-4}

The Adult Self-Report Inventory-4 (ASRI-4; Gadow et al., 2004) was used to assess Antisocial Personality Disorder (APD) symptoms as defined in the Diagnostic and Statistical Manual of Mental Disorders (5th ed.; DSM-5; American Psychiatric Association, 2013). Items on the ASRI-4 are rated on a 4-point Likert-type scale ranging from 0 ("never") to 3 ("very often"). The 7 items of the scale were summed to create an overall APD score $(\alpha=.73)$. Research with clinical and community samples has demonstrated that scores on the ASRI-4 show convergent and discriminant validity (Gadow et al., 2004; Kyranides et al., 2017).

\section{Triarchic Psychopathy Measure}

The Triarchic Psychopathy Measure (TriPM; Patrick et al., 2010) was used to assess psychopathic (PSY) traits. The TriPM is a 58-item self-report measure with items rated on a 4-point Likert-type scale ranging from 0 ("False") to 3 ("True"). The scale includes three distinct but related personality dispositions: boldness (i.e., relative fearlessness, low stress reactivity, and social dominance), meanness (i.e., deficient empathy, cruelty, and exploitation of others), and disinhibition (e.g., deficient impulse control). For the purpose of this study, the total score was used, which demonstrated adequate internal consistency in the current study $(\alpha=.88)$. Prior work has verified the validity of the TriPM in community samples (Fanti et al., 2016; Kyranides et al., 2016).

\section{Facial Task}

Dynamic stimuli portraying prototypical facial expressions were presented through an E-prime script. The stimuli featured Ekman's (1993) basic emotions of happiness, sadness, anger, fear, and pain in addition to neutral facial expressions, all of which have been used in prior work (Kyranides et al., 2020). The 1-s clips featured trained actors (4 women and 4 men) who performed the 6 expressions, resulting in the presentation of 48 stimuli in total. The task included three blocks that implicated different instructions with 16 trials presented in each block. The conditions were: a) imitation (participants were asked to imitate the featured prototypical expressions), b) suppression (participants were asked to suppress any facial responses evoked by the stimuli), and c) neutral (participants were not instructed to do anything, just provide a response). The order of condition blocks and affective stimuli was randomized to cancel any order effects. 


\section{FaceReader}

Video recordings of participants' facial expressions were edited offline in order to analyse only their facial reactions during the presentation of stimuli. For each participant, the edited recordings were uploaded into FaceReader 8.0 (Noldus Information Technology, Wageningen, The Netherlands) and analysed using batch analysis. FaceReader is a facial coding software that compares an individual's face to a database of images and classifies facial expressions into six basic emotions: angry, sad, fearful, surprised, disgusted, and happy as well as neutral. In this study, we are presenting the findings for the four emotions (angry, sad, fearful, and happy), which were related to the stimuli we used. The software identifies the participant's facial features in the digital video files and uses locations of 500 key points to make a three-dimensional reconstruction of the face. FaceReader produces a numerical output that rates the intensity of the emotional expression on a scale from 0 ("not present") to 1 ("maximum intensity"). For each recording, the numerical output was used to identify the point of highest intensity for each emotion. The software indicated that the majority of recordings analysed were of optimal quality (ranged from .91-.92). FaceReader has an accuracy of 88-89\% (Lewinski et al., 2014) and has been effectively used in other studies (e.g., Fanti et al., 2017).

\section{Plan of Analysis}

Data analysis was carried out in IBM SPSS 25. First, to identify if the groups under investigation were differentiated based on their scores on APD symptoms and psychopathic traits, an analysis of variance (ANOVA) was conducted with the groups formed (low risk, APD-only, PSY-only, and the combined APD+PSY) as the between-subject variable using the scores on APD and PSY as the outcome variables. Secondly, to examine how the conditions implemented during the task affected facial expressions, separate mixed ANOVAs were conducted with the three conditions (imitation, suppression, and neutral) and the six facial expressions to be identified (anger, fear, happy, neutral, pain, and sad) as the within-subject variables and the groups (low risk, APD-only, PSY-only, and the combined APD + PSY) as the betweensubject variable using the four FaceReader outputs (angry, sad, fearful, and happy) for the different facial expressions recorded. Finally, to examine if the instructions presented during the task impacted participants' accuracy, an additional mixed ANOVA was conducted using accuracy as the output variable. In the case that no interaction was identified, main effects are reported. Greenhouse-Geisser corrected effects, partial eta square values $\left(\eta^{2}=.01-.06\right.$ small effect size, $\eta^{2}=.06-.14$ medium effect size, $\eta^{2}>.14$ large effect size; Cohen, 1988), are reported in the text. Significant interactions are depicted in figures along with $95 \%$ confidence intervals. Post-hoc Bonferroni tests were used in all analyses.

\section{Results}

\section{Groups}

The findings from the ANOVA regarding APD scores suggested that there were significant differences between the groups, $F(3,103)=85.38, p<.001$, with the combined APD + PSY group $(M=4.26, S E=.20)$ and the APD-only group ( $M=3.94, S E=.23)$ scoring higher than the PSY-only group $(M=1.57, S E=.21)$ and the low risk group $(M=.89$, $S E=.14)$. The difference between the combined APD + PSY group and the APD-only group was not significant $(p=1.00)$. The difference between the low-risk group and the PSYonly group was also non-significant $(p=.05)$. Scores are presented in z-sores in Fig. 1. Similarly, the findings from the ANOVA regarding psychopathic scores suggested that there were significant differences between the groups, $F(3$, $103)=58.43, p<.001$, with the combined APD + PSY group $(M=64.61, S E=1.78)$ and the PSY-only group $(M=58.48$, $S E=1.86)$ scoring higher on psychopathic traits than the
Fig. 1 Identified group scores based on antisocial personality disorder symptoms and psychopathic traits scores.Zscores are shown for the different groups identified based on Antisocial Personality Disorder (APD) symptoms assessed by the Adult SelfReport Inventory-4 and psychopathic (PSY) traits scores assessed by the Triarchic Psychopathy Measure (bars represent $95 \%$ confidence intervals)

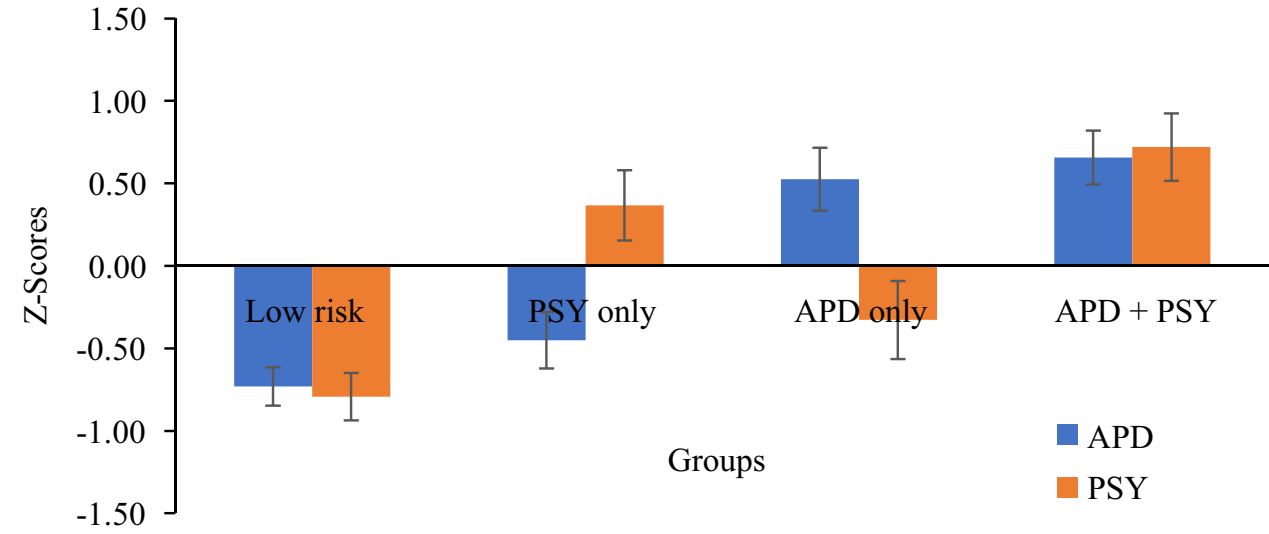


APD-only group ( $M=46.47, S E=2.07)$, which also differed to the low risk group $(M=38.41, S E=1.26)$ who reported the lowest scores. The mean scores were comparable to those reported in other community samples using similar approaches to identify subtypes at differential risk (Gadow et al., 2004; Kyranides et al., 2016, 2017; van Dongen et al., 2017).

\section{Facial Reactions Assessed Using FaceReader}

\section{Angry Facial Reactions}

The findings from the repeated measures ANOVA regarding angry facial reactions suggested that there was a main effect of group, $F(3,103)=3.25, p<.05, \eta^{2}=.09$, with the combined APD + PSY group expressing more anger $(M=.165, S E=.026)$ compared to the PSY-only group $(M=.063, S E=.028 ; p<.05)$ as shown in Fig. 2. The difference between the APD + PSY group and the APD-only group $(M=.059, S E=.03)$ was marginally significant $(p=.06)$. None of the other differences between groups were significant. A main effect for experimental condition, $F(1.95,200.88)=4.40, p<.05, \eta^{2}=.04$, was also identified. Post-hoc comparisons suggested that angry facial reactions were more intense in the imitation condition $(M=.104, S E=.014)$ compared to the suppression $(M=.083, S E=.014)$ condition $(p<.05)$ but did not differ from the neutral $(M=.096, S E=.014)$ condition $(p=1.00)$. Angry expressiveness did not differ in the neutral and suppression conditions $(p=.12)$. A main effect for emotions, $F(4.47,459.89)=13.83, p<.001$, $\eta^{2}=.118$, was also identified, indicating that, overall, participants expressed more anger in reaction to angry facial expressions $(M=.123, S E=.015)$ compared to all other stimuli presented (sad: $M=.097, S E=.015$; pain: $M=.093, S E=.014$; fear: $M=.087, S E=.013$; neutral: $M=.085, S E=.013$; and happy: $M=.081, S E=.012$; all $p$ s <.05). In addition, a significant condition by emotion interaction was found, $F(8.03,827.32)=9.00, p<.001$, $\eta^{2}=.08$, suggesting that more anger was expressed when presented with angry facial stimuli in the imitation condition compared to the other two conditions (supplementary material 1).

\section{Sad Facial Reactions}

The findings from the repeated measures ANOVA regarding sad facial reactions suggested that there was a main effect for group, $F(3,103)=2.85, p<.05, \eta^{2}=.08$, with the APDonly group expressing more sadness $(M=.064, S E=.013)$ compared to the combined APD + PSY group $(M=.014$, $S E=.011 ; p<.05)$ as shown in Fig. 3. None of the other differences between groups were significant $(p s>.05)$. There was also a main effect for experimental condition, $F(1.85$, $190.73)=5.48, p<.01, \eta^{2}=.05$, with participants expressing more sadness in the imitation condition $(M=.042, S E=.007)$ compared to both the suppression $(M=.031, S E=.006 ; p<.05)$ and neutral $(M=.031, S E=.006 ; p<.05)$ conditions, with the last two conditions not differing between them $(p=1.00)$. A main effect for emotions, $F(4.03,415.29)=10.89, p<.001$, $\eta^{2}=.096$, was also identified indicating that participants expressed more sadness when presented with sad facial expressions $(M=.050, S E=.007 ; 95 \% \mathrm{CI}[0.04,0.06])$ compared to all other stimuli presented (neutral: $M=.035, S E=.007$; fear: $M=.033, S E=.006$; anger: $M=.032, S E=.006$; pain: $M=.030$, $S E=.005$; and happy: $M=.027, S E=.005$; all $p s<.05$ ). In addition, a significant condition by emotion interaction was found, $F(5.27,542.25)=9.45, p<.001, \eta^{2}=.08$, suggesting that more sadness was expressed when presented with sad facial stimuli in the imitation condition compared to the suppression and neutral conditions (supplementary material 2).
Fig. 2 Group effect for angry facial reactions assessed with FaceReader. Angry facial expressiveness is shown for the different groups. APD $=$ Antisocial Personality Disorder symptoms; PSY $=$ Psychopathic personality traits (bars represent 95\% confidence intervals)

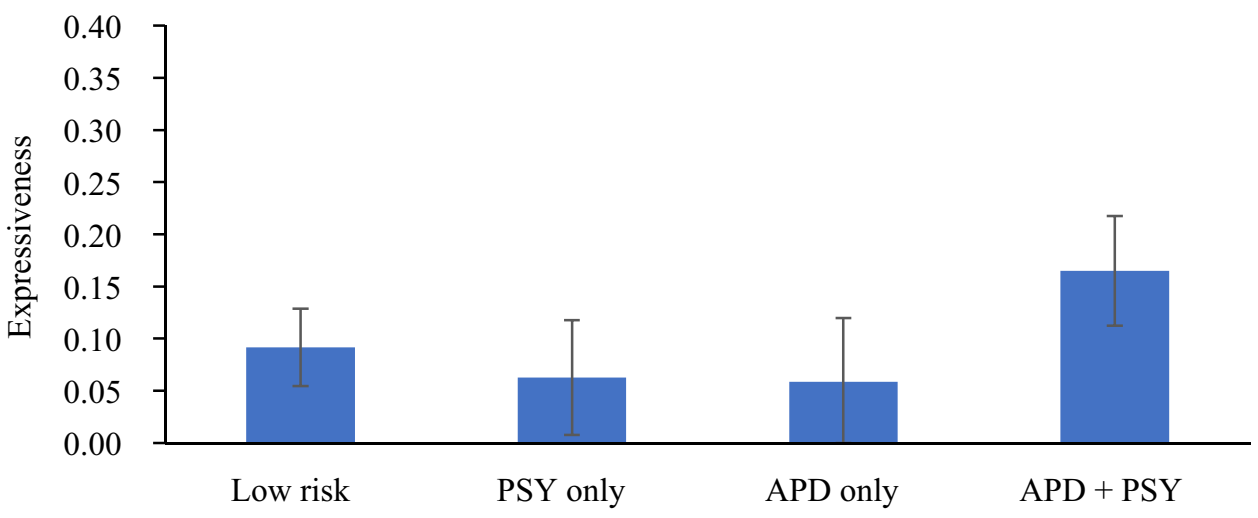

Groups 
Fig. 3 Group effect for sad facial reactions assessed with FaceReader. Sad facial expressiveness is shown for the different groups. $\mathrm{APD}=$ Antisocial Personality Disorder; PSY = Psychopathic personality traits (bars represent 95\% confidence intervals)

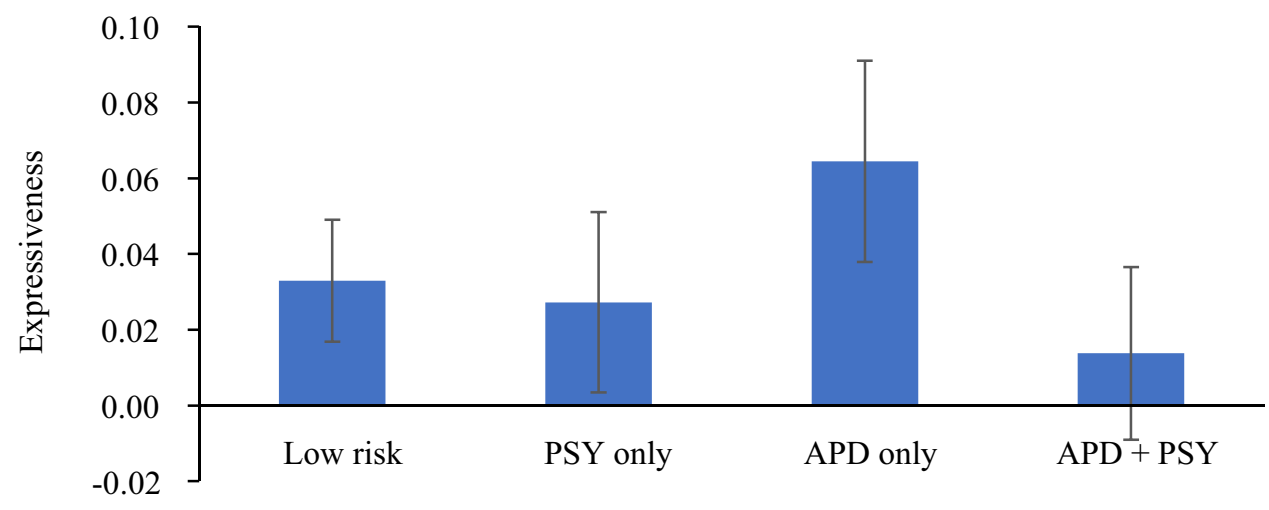

Groups

\section{Fearful Facial Reactions}

The findings from the repeated measures ANOVA regarding fearful facial reactions suggested that there was a main effect for experimental condition, $F(1.46,150.36)=10.85$, $p<.001, \eta^{2}=.095$. Post-hoc comparisons suggest that fearful facial reactions were more pronounced in the imitation condition $(M=.019, S E=.004)$ compared to both the suppression $(M=.009, S E=.002)$ and neutral $(M=.008$, $S E=.002)$ conditions $(p s<.01)$, with the last two conditions not differing between them $(p=1.00)$. A main effect for emotions, $F(2.57,264.28)=5.47, p<.01, \eta^{2}=.05$, was also identified, suggesting that participants expressed more fear when presented with stimuli that depicted fearful $(M=.014$, $S E=.003)$ and painful $(M=.017, S E=.003)$ facial expressions compared to other stimuli presented (sad: $M=.012$, $S E=.003$; angry: $M=.009, S E=.002$; neutral: $M=.010$, $S E=.003$; and happy: $M=.010, S E=.002)$. In addition, a significant condition by emotion interaction was found, $F(4.53,466.29)=5.77, p<.001, \eta^{2}=.05$, suggesting more fearful expressions were recorded when presented with fearful and painful facial stimuli in the imitation condition compared to the other two conditions (supplementary material $3)$.

\section{Happy Facial Reactions}

The findings from the repeated measures ANOVA regarding happy facial reactions as was assessed by FaceReader suggested that there was a main effect for experimental condition, $F(1.39,143.23)=44.78, p<.001, \eta^{2}=.30$, suggesting that happy facial reactions were more pronounced in the imitation condition $(M=.056, S E=.007)$ compared to both the suppression $(M=.020, S E=.004)$ and neutral $(M=.019, S E=.005)$ conditions $(p s<.001)$, with the last two conditions showing no significant difference between them $(p=1.00)$. A main effect for emotions, $F(2.31$, $238.36)=46.16, p<.001, \eta^{2}=.31$, was also identified.
Post-hoc comparisons indicated that participants expressed more happy facial reactions $(M=.074, S E=.008)$ when identifying happy facial expressions compared to all other stimuli presented (pain: $M=.036, S E=.006$; fear: $M=.022$, $S E=.004$; angry: $M=.021, S E=.004$, sad: $M=.021$, $S E=.004$; and neutral: $M=.018, S E=.004$; all $p s<.001$ ). In addition, a significant condition by emotion interaction was identified, $F(2.94,303.35)=44.45, p<.001, \eta^{2}=.30$, suggesting that more happy expressions were recorded when presented with happy and painful facial stimuli in the imitation condition compared to the other two conditions (supplementary material 4).

Overall, findings from the facial expressions assessed by FaceReader suggest that the stimuli used in the task produced the expected facial reactions. Additionally, the emotion by condition interactions identified verify that, irrespective of subgroup, participants did imitate when they were instructed to do so, as shown by an increase in expression of the emotion (recorded by FaceReader) that was being presented to them.

\section{Accuracy}

Findings from the repeated measures ANOVA suggested that there was a main effect for experimental condition, $F(1.95$, $200.55)=6.62, p<.01, \eta^{2}=.06$, with participants showing improved accuracy in the imitation condition $(M=86.61$, $S E=1.11)$ compared to both the neutral $(M=82.62$, $S E=1.15)$ and suppression $(M=82.26, S E=1.07)$ conditions ( $p \mathrm{~s}<.05$ ), with the last two conditions not differing between them $(p=1.00)$. In addition, a significant condition by group interaction was found, $F(5.84,200.55)=2.59$, $p<.05, \eta^{2}=.07$ (Fig. 4). When examining the differences in accuracy between groups in the different conditions separately, differences were significant only in the neutral condition, $F(3,106)=4.91, p<.01$, with the control group showing higher accuracy $(M=87.56, S E=1.48)$ compared to the APD+PSY $(M=78.54, S E=2.28 ; p<.01)$ and the 
Fig. 4 Accuracy scores for the different conditions and groups. $\mathrm{APD}=$ Antisocial Personality Disorder symptoms; PSY = Psychopathic personality traits (bars represent $95 \%$ confidence intervals)

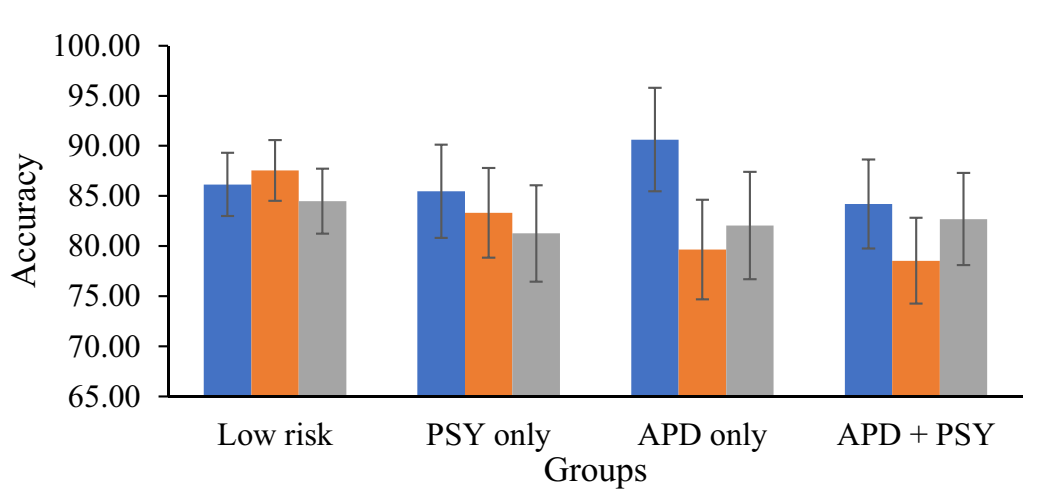

Imitation

- Neutral

- Suppression
APD-only group $(M=79.65, S E=2.91 ; p<.05)$, but no difference was found when comparing their accuracy scores with the PSY-only group $(M=83.31, S E=1.90)$. No differences were identified between the groups in the imitation or suppression conditions. When examining the differences between the conditions in the different groups separately, the APD-only group showed differences between the three conditions, $\left.F(2,32)=4.99, p<.05, \eta^{2}=.238\right)$, with improved accuracy in the imitation condition $(M=90.62, S E=2.61)$ compared to the other two experimental conditions, suppression $(M=82.05, S E=2.70 ; p<.05)$ and neutral $(M=79.65$, $S E=2.51 ; p<.05)$, but the difference between the neutral and suppression conditions was not significant $(p=1.00)$. Additionally, accuracy for the APD + PSY group was different in the three conditions, $F(2,32)=4.99, p<.05, \eta^{2}=.238$, with higher accuracy recorded in the imitation condition $(M=84.20, S E=2.31)$ compared to the neutral condition $(M=78.54, S E=2.29 ; p<.05)$ but not the suppression condition $(M=82.70, S E=2.10 ; p=1.00)$. A main effect for emotions, $F(4.12,424.54)=28.42, p<.001, \eta^{2}=.228$, was also identified, indicating that participants showed higher accuracy when identifying happy $(M=96.21, S E=.88)$ and angry $(M=91.51, S E=1.40)$ facial expressions, followed by fear $(M=83.45, S E=1.76)$ and $\operatorname{sad}(M=82.00, S E=1.64)$ facial expressions and were less accurate in identifying neutral $(M=76.08, S E=1.92)$ and expressions depicting pain $(M=73.76, S E=2.23)$.

\section{Discussion}

The current study examined how young adults with different levels of APD symptoms and psychopathic traits process emotional facial expressions across different experimental conditions (imitation, suppression, and neutral). This study is, to the best of our knowledge, the first to use facial video analysis software to examine the effect of an instruction to imitate on participants' facial reactions to negative and positive facial affective cues. Overall, results from this study were partially consistent with our hypotheses. Emotion recognition accuracy was significantly lower among the group with co-occurring psychopathic traits and antisocial behavior (APD+ PSY) compared to the control group in the neutral condition when no instructions were provided. The combined group (APD + PSY) showed increased angry facial expressions compared to the other groups, but, surprisingly, the APD-only group showed more pronounced sad facial expressions during the imitation condition. Moreover, as predicted, the instruction to imitate affective cues had an overall positive effect on participants' facial emotion recognition accuracy. Further analysis suggested that these improvements in accuracy in the imitation condition compared to the neutral condition were more pronounced in the APD-only group and less pronounced in the APD + PSY group. Finally, the study has methodological implications since the FaceReader software was deemed effective in assessing facial reactivity, as participants produced the expected facial reactions for each emotional stimulus presented when instructed to do so (i.e., in the imitation condition and not in the other two conditions, suppression and neutral).

The current findings are aligned with prior work documenting deficits in facial emotion recognition in individuals with psychopathic traits and antisocial behavior (Marsh \& Blair, 2008; Rogstad \& Rogers, 2008). In the present study, the APD + PSY group and the APD-only group both had significantly lower accuracy ratings in the neutral condition than the control group. Prior work suggests similar findings with the group with co-occurring antisocial behavior and psychopathic traits (APD+PSY) reporting more severe deficits in emotion processing compared to individuals showing elevated psychopathic traits without behavioral problems (PSY-only) (Kyranides et al., 2016; Marsden et al., 2019). Interestingly, the PSY-only group showed similar performance to controls with regard to accuracy. These results align with the idea that the presence of psychopathic traits in isolation from antisocial behavior may represent the profile of so-called "successful" psychopathy, in which individuals function effectively despite limited empathy (Gao \& Raine, 2010; Kyranides et al., 2016). Our findings further indicate 
the importance of considering groups with isolated or cooccurring APD symptoms and psychopathic traits when observing deficits.

This study also compared differences between groups with varying profiles of APD symptoms and psychopathic traits in expressivity in response to facial affective stimuli. The APD + PSY group expressed more anger compared to the PSY-only group and marginally more anger than the APD-only group, which suggests that individuals with comorbid antisocial behavior and psychopathic traits tend to express more anger when exposed to angry facial expressions or in response to affective stimuli (Fanti et al., 2017). This is aligned with recent work (Kosson et al., 2020) showing a link between psychopathic traits and elevated levels of chronic anger expression. Surprisingly, the APD-only group was found to express more sadness compared to the APD + PSY group. This finding corresponds with prior research in which individuals with APD + PSY exhibited deficits in the recognition of sadness, while those with only APD symptoms did not (Fairchild et al., 2009). Our findings suggest that expressing sadness and correctly identifying sadness might be connected in these groups of individuals. Furthermore, identifying differences between individuals with antisocial behavior and/or psychopathic traits in the expression of specific emotions may further explain differences in how these groups regulate emotions. For example, the pronounced expression of anger in the APD + PSY group may indicate that this group reacts to affective situations with increased anger (Fanti et al., 2017). As others have suggested (e.g., Fanti et al., 2017; Frick \& Viding, 2009), groups with antisocial behavior might therefore benefit from interventions emphasizing emotion regulation such as anger management. Conversely, the limited reactions of the PSY-only group may further demonstrate that individuals with psychopathic traits in isolation (without displaying antisocial behavior) are better at regulating or controlling their emotions (Frick \& Viding, 2009). Congruent with our predictions and prior studies (Khvatskaya \& Lenzenweger, 2016; Owens et al., 2017), there were no differences in facial reactions to positive emotions in the groups with higher APD symptoms and psychopathic traits. Group differences in facial emotion expressivity further support prior work highlighting that individuals with antisocial behavior and psychopathic traits are a heterogeneous group (Marsden et al., 2019; Sarkar et al., 2011) who show differences in how they regulate their emotions and, therefore, future prevention/intervention efforts should take these differences into consideration.

Facial emotion recognition deficits in groups with APD symptoms and psychopathic traits have been the focus of previous interventions using strategies to refocus their attention (Dadds et al., 2008; Kyranides et al., 2020), and such efforts have proven effective at improving facial emotion recognition accuracy. The present study aimed to shed more light on this area and used an experimental manipulation asking participants to imitate facial expressions before identifying them, which has proven effective in improving facial emotion recognition in the groups with the largest deficits. Similar findings have been reported with other samples with similar deficits such as those on the autism spectrum (Lewis \& Dunn, 2017). Participants in our study did comply with the instructions of the task as evidenced by their increased expressivity for the different stimuli presented in the imitation condition. The instruction to imitate facial expressions also improved accuracy for the APD-only group and for the APD+PSY group compared to when no instructions were provided. The instruction to imitate may have had little effect on the PSY-only group because this group did not have significant deficits to begin with, as they showed similar accuracy ratings to the control group. By asking participants to imitate facial expressions, this study attempted not only to draw participants' attention to various facial features but also to induce facial mimicry and thereby promote emotional contagion (Prochazkova \& Kret, 2017). Since emotions involve behavioral, physiological, and cognitive components, activation of one component automatically activates other components (Wood et al., 2016), and findings from FaceReader outputs suggest that, at least on a behavioral level, this was achieved. The current findings are encouraging for efforts focused on improving emotion recognition deficits by targeting attention mechanisms and emotional contagion and suggest that this can be achieved with promising results for individuals with predominantly behavioral symptoms.

FaceReader was effective in correctly identifying the emotions being expressed. The study revealed variations in emotional processes between groups and experimental conditions (imitation vs. neutral and suppression) as well as the stimuli being presented, which confirmed that FaceReader was able to identify subtle changes in facial reactions. Within the same field of research, Fanti et al. (2017) showed that FaceReader findings are aligned with fEMG data. Similarly, other studies found high correlations between fEMG and FaceReader software for angry and happy expressions (Kulke et al., 2020) and argue that FaceReader is a reliable measuring tool (Skiendziel et al., 2019) able to detect these micro reactions.

The current findings should be interpreted considering some limitations. First, the study was based on a community sample and therefore a replication of the findings in a clinical sample with higher levels of APD symptoms and psychopathic traits is warranted. Secondly, facial expressivity was assessed using FaceReader, however, future studies should use a combination of measures to do this including fEMG. Thirdly, the sample consisted mainly of female participants. Anton et al. (2012) found that males and females 
with psychopathic traits and APD symptoms exhibit differences in perceiving, interpreting, and reacting to emotion cues, so future research should aim to recruit a more gender balanced sample. Lastly, impairments in emotion processing and empathy, albeit with different etiologies, are also present in other conditions such as autism spectrum disorders (Bird \& Viding, 2014). Future studies may want to consider controlling for autistic traits in their samples. Despite these limitations, this study has several strengths, including the fact that a large sample of participants was screened beforehand. It allowed us to identify the individuals with the different profiles of interest. Moreover, the facial stimuli used in the current study were more realistic and ecologically valid compared to static pictures used in past studies (Herpertz et al., 2001). Finally, a more objective measure was used to assess participants' expressivity and adherence to experimental instructions (FaceReader).

Overall, the study offers a novel contribution to the literature by assessing facial mimicry as a way to investigate emotion processing differences between individuals with varying levels of APD symptoms and psychopathic traits. Findings highlight how symptoms of APD, psychopathic traits, and a combination of the two are associated with different deficits in emotion processing and reactivity. Individuals with higher levels of APD symptoms and psychopathic traits expressed more anger, whereas individuals with higher levels of APD symptoms and low psychopathic traits (APD-only group) expressed more sadness. This is the first study to our knowledge to assess the effectiveness of facial mimicry in improving facial emotion recognition accuracy by measuring facial reactions using FaceReader. The imitation instruction seems to be effective in improving accuracy for individuals with APD symptoms with or without psychopathic traits but is more effective for those without, suggesting that the capacity for typical facial emotion recognition may be present in this group when attention is properly directed. However, further research is needed to see if these effects are long-lasting and to determine which components of emotional processing are being targeted by an instruction to imitate (attention and/or motor activity). The current findings highlight the heterogeneity of this population and show the importance of tailoring future research to the profile of the individual by considering underlying mechanisms.

Supplementary Information The online version contains supplementary material available at https://doi.org/10.1007/s12144-022-02749-0.

Authors' Contributions The corresponding author presented the idea and supervised the findings of this work. All authors discussed the results and contributed to the final manuscript.

Data Availability The datasets generated during and/or analysed during the current study are available from the corresponding author on reasonable request.
Code Availability Output of analysis conducted is available from the corresponding author on reasonable request.

\section{Declarations}

Ethical Approval All procedures performed in the study involving human participants were in accordance with the ethical standards of the institutional and national research committee and with the 1964 Helsinki declaration and its later amendments or comparable ethical standards.

Consent to Participate Informed consent was obtained from all individual participants included in the study.

Consent for Publication No identifiable information was collected.

Conflicts of Interest/Competing Interests Authors have no conflicts of interest to disclose.

Open Access This article is licensed under a Creative Commons Attribution 4.0 International License, which permits use, sharing, adaptation, distribution and reproduction in any medium or format, as long as you give appropriate credit to the original author(s) and the source, provide a link to the Creative Commons licence, and indicate if changes were made. The images or other third party material in this article are included in the article's Creative Commons licence, unless indicated otherwise in a credit line to the material. If material is not included in the article's Creative Commons licence and your intended use is not permitted by statutory regulation or exceeds the permitted use, you will need to obtain permission directly from the copyright holder. To view a copy of this licence, visit http://creativecommons.org/licenses/by/4.0/.

\section{References}

American Psychiatric Association, DSM-5 Task Force. (2013). Diagnostic and statistical manual of mental disorders: DSM-5TM (5th ed.). American Psychiatric Publishing, Inc.. https://doi.org/10. 1176/appi.books.9780890425596

Anton, M. E., Baskin-Sommers, A. R., Vitale, J. E., Curtin, J. J., \& Newman, J. P. (2012). Differential effects of psychopathy and antisocial personality disorder symptoms on cognitive and fear processing in female offenders. Cognitive, Affective, \& Behavioral Neuroscience, 12(4), 761-776. https://doi.org/10.3758/ s13415-012-0114-x

Bagcioglu, E., Isikli, H., Demirel, H., Sahin, E., Kandemir, E., Dursun, P., Yuksek, E., \& Emul, M. (2014). Facial emotion recognition in male antisocial personality disorders with or without adult attention deficit hyperactivity disorder. Comprehensive Psychiatry, 55(5), 1152-1156. https://doi.org/10.1016/j.comppsych.2014. 02.011

Baskin-Sommers, A., Curtin, J. J., Li, W., \& Newman, J. P. (2012). Psychopathy-related differences in selective attention are captured by an early event-related potential. Personality Disorders: Theory, Research, and Treatment, 3(4), 370-378. https://doi.org/10.1037/ a0025593

Bird, G., \& Viding, E. (2014). The self to other model of empathy: Providing a new framework for understanding empathy impairments in psychopathy, autism, and alexithymia. Neuroscience and Biobehavioral Reviews, 47, 520-532. https://doi.org/10.1016/j. neubiorev.2014.09.021 
Blair, R. J. R. (2013). Psychopathy: Cognitive and neural dysfunction. Dialogues in Clinical Neuroscience, 15(2), 181-190. https://doi. org/10.31887/DCNS.2013.15.2/rblair

Blair, R. J. R. (2018). Traits of empathy and anger: Implications for psychopathy and other disorders associated with aggression. Philosophical Transactions of the Royal Society B: Biological Sciences, 373(1744), 20170155. https://doi.org/10.1098/rstb. 2017.0155

Book, A., Methot, T., Gauthier, N., Hosker-Field, A., Forth, A., Quinsey, V., \& Molnar, D. (2015). The mask of sanity revisited: Psychopathic traits and affective mimicry. Evolutionary Psychological Science, 1(2), 91-102. https://doi.org/10.1007/ s40806-015-0012-x

Chartrand, T. L., \& Bargh, J. A. (1999). The chameleon effect: The perception-behavior link and social interaction. Journal of Personality and Social Psychology, 76(6), 893-910. https://doi.org/ 10.1037/0022-3514.76.6.893

Chialant, D., Edersheim, J., \& Price, B. H. (2016). The dialectic between empathy and violence: An opportunity for intervention? The Journal of Neuropsychiatry and Clinical Neurosciences, 28(4), 273-285. https://doi.org/10.1176/appi.neuro psych. 15080207

Conson, M., Ponari, M., Monteforte, E., Ricciato, G., Sarà, M., Grossi, D., \& Trojano, L. (2013). Explicit recognition of emotional facial expressions is shaped by expertise: Evidence from professional actors. Frontiers in Psychology, 4(382), 1-8. https://doi.org/10.3389/fpsyg.2013.00382

Crego, C., \& Widiger, T. A. (2015). Psychopathy and the DSM. Journal of Personality, 83(6), 665-677. https://doi.org/10.1111/ jopy. 12115

Dadds, M. R., El Masry, Y., Wimalaweera, S., \& Guastella, A. J. (2008). Reduced eye gaze explains "fear blindness" in childhood psychopathic traits. Journal of the American Academy of Child and Adolescent Psychiatry, 47(4), 455-463. https://doi.org/10. 1097/CHI.0b013e31816407f1

Dapretto, M., Davies, M. S., Pfeifer, J. H., Scott, A. A., Sigman, M., Bookheimer, S. Y., \& Iacoboni, M. (2006). Understanding emotions in others: Mirror neuron dysfunction in children with autism spectrum disorders. Nature Neuroscience, 9(1), 28-30. https://doi.org/10.1038/nn1611

Dawel, A., O'Kearney, R., McKone, E., \& Palermo, R. (2012). Not just fear and sadness: Meta-analytic evidence of pervasive emotion recognition deficits for facial and vocal expressions in psychopathy. Neuroscience \& Biobehavioral Reviews, 36(10), 2288-2304. https://doi.org/10.1016/j.neubiorev.2012.08.006

de Wied, M., van Boxtel, A., Zaalberg, R., Goudena, P. P., \& Matthys, W. (2006). Facial EMG responses to dynamic emotional facial expressions in boys with disruptive behavior disorders. Journal of Psychiatric Research, 40(2), 112-121. https://doi. org/10.1016/j.jpsychires.2005.08.003

de Wied, M., van Boxtel, A., Matthys, W., \& Meeus, W. (2012). Verbal, facial and autonomic responses to empathy-eliciting film clips by disruptive male adolescents with high versus low callous-unemotional traits. Journal of Abnormal Child Psychology, 40(2), 211-223. https://doi.org/10.1007/s10802-011-9557-8

Ekman, P. (1993). Facial expression and emotion. American Psychologist, 48(4), 384-392. https://doi.org/10.1037/0003-066x. 48.4.384

Fairchild, G., Van Goozen, S. H., Calder, A. J., Stollery, S. J., \& Goodyer, I. M. (2009). Deficits in facial expression recognition in male adolescents with early-onset or adolescence-onset conduct disorder. Journal of Child Psychology and Psychiatry, and Allied Disciplines, 50(5), 627-636. https://doi.org/10.1111/j.1469-7610. 2008.02020.x

Fanti, K. A., Kyranides, M. N., Drislane, L. E., Colins, O. F., \& Andershed, H. (2016). Validation of the greek cypriot translation of the
Triarchic Psychopathy Measure. Journal of Personality Assessment, 98(2), 146-154. https://doi.org/10.1080/00223891.2015. 1077452

Fanti, K. A., Kyranides, M. N., \& Panayiotou, G. (2017). Facial reactions to violent and comedy films: Association with callous-unemotional traits and impulsive aggression. Cognition \& Emotion, 31(2), 209-224. https://doi.org/10.1080/02699931.2015.1090958

Fecteau, S., Pascual-Leone, A., \& Théoret, H. (2008). Psychopathy and the mirror neuron system: Preliminary findings from a nonpsychiatric sample. Psychiatry Research, 160(2), 137-144. https:// doi.org/10.1016/j.psychres.2007.08.022

Frick, P. J., \& Viding, E. (2009). Antisocial behavior from a developmental psychopathology perspective. Development and Psychopathology, 21(4), 1111-1131. https://doi.org/10.1017/S0954 579409990071

Gadow, K. D., Sprafkin, J., \& Weiss, M. (2004). Adult self-report Inventory-4 manual. Stony Brook.

Gao, Y., \& Raine, A. (2010). Successful and unsuccessful psychopaths: a neurobiological model. Behavioral Sciences \& The Law, 28(2), 194-210. https://doi.org/10.1002/bsl.924

Gazzola, V., Aziz-Zadeh, L., \& Keysers, C. (2006). Empathy and the somatotopic auditory mirror system in humans. Current Biology, 16(18), 1824-1829. https://doi.org/10.1016/j.cub.2006.07.072

Gehrer, N. A., Duchowski, A. T., Jusyte, A., \& Schönenberg, M. (2020). Eye contact during live social interaction in incarcerated psychopathic offenders. Personality Disorders: Theory, Research, and Treatment, 11(6), 431-439. https://doi.org/10.1037/per00 00400

Gillespie, S. M., Rotshtein, P., Wells, L. J., Beech, A. R., \& Mitchell, I. J. (2015). Psychopathic traits are associated with reduced attention to the eyes of emotional faces among adult male non-offenders. Frontiers in Human Neuroscience, 9, 552. https://doi.org/10.3389/ fnhum.2015.00552

Goldman, A. I., \& Sripada, C. S. (2005). Simulationist models of facebased emotion recognition. Cognition, 94(3), 193-213. https://doi. org/10.1016/j.cognition.2004.01.005

Habel, U., Kühn, E., Salloum, J. B., Devos, H., \& Schneider, F. (2002). Emotional processing in psychopathic personality. Aggressive Behavior, 28(5), 394-400. https://doi.org/10.1002/ab.80015

Herpertz, S. C., Werth, U., Lukas, G., Qunaibi, M., Schuerkens, A., Kunert, H. J., Freese, R., Flesch, M., Mueller-Isberner, R., Osterheider, M., \& Sass, H. (2001). Emotion in criminal offenders with psychopathy and borderline personality disorder. Archives of General Psychiatry, 58(8), 737-745. https://doi.org/10.1001/ archpsyc.58.8.737

Hess, U., \& Blairy, S. (2001). Facial mimicry and emotional contagion to dynamic emotional facial expressions and their influence on decoding accuracy. International Journal of Psychophysiology, 40(2), 129-141. https://doi.org/10.1016/s0167-8760(00)00161-6

Khvatskaya, Y., \& Lenzenweger, M. F. (2016). Motor empathy in individuals with psychopathic traits: A preliminary study. Journal of Personality Disorders, 30(5), 613-632. https://doi.org/10.1521/ pedi_2015_29_219

Kosson, D. S., Garofalo, C., McBride, C. K., \& Velotti, P. (2020). Get mad: Chronic anger expression and psychopathic traits in three independent samples. Journal of Criminal Justice, 67, 101672. https://doi.org/10.1016/j.jcrimjus.2020.101672

Kulke, L., Feyerabend, D., \& Schacht, A. (2020). A comparison of the affectiva iMotions facial expression analysis software with EMG for identifying facial expressions of emotion. Frontiers in Psychology, 11, 329. https://doi.org/10.3389/fpsyg.2020.00329

Künecke, J., Mokros, A., Olderbak, S., \& Wilhelm, O. (2018). Facial responsiveness of psychopaths to the emotional expressions of others. PLoS ONE, 13(1), e0190714. https://doi.org/10.1371/journ al.pone. 0190714 
Kyranides, M. N., Fanti, K. A., \& Panayiotou, G. (2016). The disruptive adolescent as a grown-up: Predicting adult startle responses to violent and erotic films from adolescent conduct problems and callous-unemotional traits. Journal of Psychopathology and Behavioral Assessment, 38(2), 183-194. https://doi.org/10.1007/ s10862-015-9520-z

Kyranides, M. N., Fanti, K. A., Sikki, M., \& Patrick, C. J. (2017). Triarchic dimensions of psychopathy in young adulthood: Associations with clinical and physiological measures after accounting for adolescent psychopathic traits. Personality Disorders: Theory, Research, and Treatment, 8(2), 140-149. https://doi.org/10.1037/ per0000193

Kyranides, M. N., Fanti, K. A., Petridou, M., \& Kimonis, E. R. (2020). In the eyes of the beholder: Investigating the effect of visual probing on accuracy and gaze fixations when attending to facial expressions among primary and secondary callous-unemotional variants. European Child \& Adolescent Psychiatry, 29(10), 1441-1451. https://doi.org/10.1007/ s00787-019-01452-z

Lee, D. H., \& Anderson, A. K. (2017). Reading what the mind thinks from how the eye sees. Psychological Science, 28(4), 494-503. https://doi.org/10.1177/0956797616687364

Lewinski, P., den Uyl, T. M., \& Butler, C. (2014). Automated facial coding: Validation of basic emotions and FACS AUs in FaceReader. Journal of Neuroscience, Psychology, and Economics, 7(4), 227-236. https://doi.org/10.1037/npe0000028

Lewis, M. B., \& Dunn, E. (2017). Instructions to mimic improve facial emotion recognition in people with sub-clinical autism traits. Quarterly Journal of Experimental Psychology, 70(11), 2357-2370. https://doi.org/10.1080/17470218.2016.1238950

Lockwood, P. L. (2016). The anatomy of empathy: Vicarious experience and disorders of social cognition. Behavioural Brain Research, 311, 255-266. https://doi.org/10.1016/j.bbr.2016. 05.048

MacCoon, D. G., Wallace, J. F., \& Newman, J. P. (2004). Self-regulation: Context-appropriate balanced attention. In Handbook of self-regulation: Research, theory, and applications (pp. 422-444). The Guilford Press.

Marsden, J., Glazebrook, C., Tully, R., \& Völlm, B. (2019). Do adult males with antisocial personality disorder (with and without comorbid psychopathy) have deficits in emotion processing and empathy? A systematic review. Aggression and Violent Behavior, 48, 197-217. https://doi.org/10.1016/j.avb.2019.08.009

Marsh, A. A., \& Blair, R. J. (2008). Deficits in facial affect recognition among antisocial populations: A meta-analysis. Neuroscience and Biobehavioral Reviews, 32(3), 454-465. https://doi. org/10.1016/j.neubiorev.2007.08.003

Meffert, H., Gazzola, V., den Boer, J. A., Bartels, A. A., \& Keysers, C. (2013). Reduced spontaneous but relatively normal deliberate vicarious representations in psychopathy. Brain: A Journal of Neurology, 136(8), 2550-2562. https://doi.org/10.1093/brain/awt190

Neal, D. T., \& Chartrand, T. L. (2011). Embodied emotion perception: Amplifying and dampening facial feedback modulates emotion perception accuracy. Social Psychological and Personality Science, 2(6), 673-678. https://doi.org/10.1177/19485 50611406138

Newman, J. P., Curtin, J. J., Bertsch, J. D., \& Baskin-Sommers, A. R. (2010). Attention moderates the fearlessness of psychopathic offenders. Biological Psychiatry, 67(1), 66-70. https://doi.org/ 10.1016/j.biopsych.2009.07.035

Niedenthal, P. M., Halberstadt, J. B., Margolin, J., \& Innes-Ker, A. H. (2000). Emotional state and the detection of change in facial expression of emotion. European Journal of Social Psychology, $30(2), 211-222$.

Oberman, L. M., Winkielman, P., \& Ramachandran, V. S. (2007). Face to face: Blocking facial mimicry can selectively impair recognition of emotional expressions. Social Neuroscience, 2(3-4), 167-178. https://doi.org/10.1080/17470910701391943

Owens, E. S., McPharlin, F., Brooks, N., \& Fritzon, K. (2017). The effects of empathy, emotional intelligence and psychopathy on interpersonal interactions. Psychiatry, Psychology, and Law: An Interdisciplinary Journal of the Australian and New Zealand Association of Psychiatry, Psychology and Law, 25(1), 1-18. https://doi.org/10.1080/13218719.2017.1347936

Patrick, C. J., Fowles, D. C., \& Krueger, R. F. (2010). Triarchic conceptualization of psychopathy: Developmental origins of disinhibition, boldness, and meanness. Development and Psychopathology, 21(3), 913-938. https://doi.org/10.1017/s0954 579409000492

Patterson, C. M., \& Newman, J. P. (1993). Reflectivity and learning from aversive events: Toward a psychological mechanism for the syndromes of disinhibition. Psychological Review, 100(4), 716-736. https://doi.org/10.1037/0033-295x.100.4.716

Peterson, M. F., \& Eckstein, M. P. (2011). Fixating the eyes is an optimal strategy across important face (related) tasks. Journal of Vision, 11(11), 662-662. https://doi.org/10.1167/11.11.662

Prochazkova, E., \& Kret, M. E. (2017). Connecting minds and sharing emotions through mimicry: A neurocognitive model of emotional contagion. Neuroscience and Biobehavioral Reviews, 80, 99-114. https://doi.org/10.1016/j.neubiorev.2017.05.013

Riser, R. E., \& Kosson, D. S. (2013). Criminal behavior and cognitive processing in male offenders with antisocial personality disorder with and without comorbid psychopathy. Personality Disorders: Theory, Research, and Treatment, 4(4), 332-340. https://doi.org/10.1037/a0033303

Rogstad, J. E., \& Rogers, R. (2008). Gender differences in contributions of emotion to psychopathy and antisocial personality disorder. Clinical Psychology Review, 28(8), 1472-1484. https:// doi.org/10.1016/j.cpr.2008.09.004

Rymarczyk, K., Żurawski, Ł., Jankowiak-Siuda, K., \& Szatkowska, I. (2018). Neural correlates of facial mimicry: Simultaneous measurements of EMG and BOLD responses during perception of dynamic compared to static facial expressions. Frontiers in Psychology, 9, 52. https://doi.org/10.3389/fpsyg.2018.00052

Sarkar, S., Clark, B. S., \& Deeley, Q. (2011). Differences between psychopathy and other personality disorders: Evidence from neuroimaging. Advances in Psychiatric Treatment, 17(3), 191200. https://doi.org/10.1192/apt.bp.107.004747

Schiffer, B., Pawliczek, C., Müller, B. W., Wiltfang, J., Brüne, M., Forsting, M., Gizewski, E. R., Leygraf, N., \& Hodgins, S. (2017). Neural mechanisms underlying affective theory of mind in violent antisocial personality disorder and/or schizophrenia. Schizophrenia Bulletin, 43(6), 1229-1239. https://doi.org/10. 1093/schbul/sbx012

Schneider, K. G., Hempel, R. J., \& Lynch, T. R. (2013). That "poker face" just might lose you the game! The impact of expressive suppression and mimicry on sensitivity to facial expressions of emotion. Emotion, 13(5), 852-866. https://doi.org/10.1037/ a0032847

Seara-Cardoso, A., Sebastian, C. L., Viding, E., \& Roiser, J. P. (2016). Affective resonance in response to others' emotional faces varies with affective ratings and psychopathic traits in amygdala and anterior insula. Social Neuroscience, 11(2), 140 152. https://doi.org/10.1080/17470919.2015.1044672

Skiendziel, T., Rösch, A. G., \& Schultheiss, O. C. (2019). Assessing the convergent validity between the automated emotion recognition software Noldus FaceReader 7 and facial action coding system scoring. PLoS One, 14(10), e0223905. https://doi.org/ 10.1371/journal.pone.0223905

Sommerville, J. A., \& Decety, J. (2006). Weaving the fabric of social interaction: Articulating developmental psychology and cognitive neuroscience in the domain of motor cognition. 
Psychonomic Bulletin \& Review, 13(2), 179-200. https://doi. org/10.3758/bf03193831

van Dongen, J. D. M., Drislane, L. E., Nijman, H., Soe-Agnie, S. E., \& van Marle, H. J. C. (2017). Further evidence for reliability and validity of the Triarchic Psychopathy Measure in a forensic sample and a community sample. Journal of Psychopathology and Behavioral Assessment, 39(1), 58-66. https://doi.org/10. 1007/s10862-016-9567-5

Venables, N. C., Hall, J. R., \& Patrick, C. J. (2014). Differentiating psychopathy from antisocial personality disorder: A triarchic model perspective. Psychological Medicine, 44(5), 1005-1013. https://doi.org/10.1017/S003329171300161X
Verona, E., Sprague, J., \& Sadeh, N. (2012). Inhibitory control and negative emotional processing in psychopathy and antisocial personality disorder. Journal of Abnormal Psychology, 121(2), 498-510. https://doi.org/10.1037/a0025308

Wood, A., Rychlowska, M., Korb, S., \& Niedenthal, P. (2016). Fashioning the face: Sensorimotor simulation contributes to facial expression recognition. Trends in Cognitive Sciences, 20(3), 227-240. https://doi.org/10.1016/j.tics.2015.12.010

Publisher's note Springer Nature remains neutral with regard to jurisdictional claims in published maps and institutional affiliations. 